\title{
Meshless implementations of Local Integral Equations for bending of thin plates
}

\author{
V. Sladek, J. Sladek \& L. Sator \\ Institute of Construction and Architecture, Slovak Academy of Sciences, \\ Bratislava, Slovakia
}

\begin{abstract}
In this paper, we show that the decomposition of the biharmonic equation into two Poisson equations is applicable to the general case of boundary conditions and any shape of the boundary edge of the plate, if we use the Local Integral Equation (LIE) formulation and a meshless approximation for primary field variables. Moreover, the spatial dependence of the bending stiffness does not give rise to any difficulties in this formulation. Such a formulation enables us to decrease the order of the derivatives of the field variables and so to increase the accuracy of numerical solutions. The efficiency and accuracy of several formulations is discussed in numerical tests using two alternative meshless approximations.

Keywords: Kirchhoff theory, biharmonic equation, Poisson equations, mesh-free methods, field derivatives.
\end{abstract}

\section{Introduction}

It is well known that high order derivatives of field variables in the governing equations give rise to difficulties in solution of boundary value problems because of worse accuracy of numerically evaluated high order derivatives. The order of the differential operator can be decreased mathematically by decomposing this operator into two lower order differential operators with introducing new field variables. However, the formulation of the physical boundary conditions in terms of new field variables can be problematic. Basically, it is possible to express all the original boundary densities in terms of the new fields and their derivatives as long as a domain-type approximation is employed in the numerical implementation. The question is, however, if the order of the derivatives is lower 
than in the original expression of the boundary conditions. In this paper, we shall discuss the decomposition of the biharmonic equation for thin plate bending [1] into two Poisson equations. In two recent decades, solution of many engineering problems as well as problems of mathematical physics have been reformulated by using various mesh free formulations with meshless approximations belonging to domain-type approximations. It is known that in the weak formulations, it is possible to decrease the order of the derivatives of field variables by transferring the derivatives to test functions (via integrations by parts) and by appropriate selection of the test functions and their lower order derivatives as vanishing on the boundary. Basically, this can be done in the weak formulations on local sub-domains [2] with choosing simple shape of the subdomains. Recall that in this procedure, the order of the derivatives occurring in boundary conditions is not changed.

In this paper, we develop either the strong formulation or the Local Integral Equation (LIE) formulation (weak formulation) and a meshless approximation for primary field variables in decomposed problem. In the local weak formulation, the constant test function with the support on the sub-domain is utilized. For solution of the original plate bending problem, we present only the local weak formulation. The strong formulation is not considered because of the $4^{\text {th }}$ order derivatives of deflections. Two kinds of the meshless approximations are employed in this paper, such as the Moving Least Square (MLS) approximation [3] and the Point Interpolation Method (PIM) [5]. Several illustrative examples are presented, in order to compare the accuracy and convergence of numerical solutions as well as the computational efficiency of various methods.

\section{Problem formulation and physical decomposition}

In the classical Kirchhoff's theory of bending of thin plates [1], all the physical quantities can be expressed in terms of the deflection $w(\mathbf{x})$ and/or its derivatives.

For the plate of thickness $h$ and mid-plane $\Omega$ orthogonal to the $x_{3}$-axis, the governing equation is given by the biharmonic operator as

$$
D \nabla^{2} \nabla^{2} w=q,
$$

where the bending stiffness $D E h^{3} / 12\left(1-v^{2}\right)$ is assumed to be constant.

The bending moment $M$, twisting moment $T$, transversal shear force $N$ and the equivalent shear force $V$ on the boundary edge $\Gamma=\partial \Omega$ are given as

$$
\begin{gathered}
M=-D\left[(1-v) n_{i} n_{j} w_{, i j}+v \nabla^{2} w\right] \\
T=-D(1-v) n_{i} t_{j} w_{, i j}=-D(1-v) t_{i} n_{j} w_{, i j} \\
N=-n_{i} D \nabla^{2} w_{, i}, \quad V=N+\frac{\partial T}{\partial \mathbf{t}}
\end{gathered}
$$


with $n_{i}$ and $t_{i}$ being the Cartesian components of the unit normal and tangent vectors on $\Gamma$. Three basic boundary conditions can be assumed on the boundary edge $\Gamma$ :

(i) clamped edge: $\left.w\right|_{\Gamma}=0 ;\left.\frac{\partial w}{\partial n}\right|_{\Gamma}=0$

(ii) simply supported edge: $\left.w\right|_{\Gamma}=0 ;\left.\quad M\right|_{\Gamma}=0$

(iii) free edge: $\left.M\right|_{\Gamma}=0 ;\left.\quad V\right|_{\Gamma}=0$.

Since the governing equation involves the fourth order derivatives of deflections, the fourth and third order derivatives of deflections are occurring in the strong and weak formulation for solution, respectively. Thus, one can expect serious difficulties in numerical solution owing to inaccurate approximation of high order derivatives of deflections in both the discretized governing equations as well as in the boundary conditions. Therefore it is appropriate to introduce the new field variable defined as

$$
m(\mathbf{x}):=-D \nabla^{2} w(\mathbf{x}), \text { for } \mathbf{x} \in \Omega
$$
(7)

Then, the governing equation (1) is split into two equations given by (6) and

$$
\nabla^{2} m(\mathbf{x})=q(\mathbf{x}) \quad \text { for } \mathbf{x} \in \Omega .
$$

In view of the definitions (2)-(4) and (6), one obtains on the boundary edge $\Gamma$

$$
\begin{gathered}
M=v m-D(1-v) n_{i} n_{j} w_{, i j}=v m-D(1-v) \frac{\partial}{\partial \mathbf{n}} \frac{\partial w}{\partial \mathbf{n}} \\
=m+D(1-v)\left[\frac{\partial}{\partial \mathbf{t}} \frac{\partial w}{\partial \mathbf{t}}-\kappa \frac{\partial w}{\partial \mathbf{n}}\right] \\
T=-D(1-v) \frac{\partial}{\partial \mathbf{n}} \frac{\partial w}{\partial \mathbf{t}}=-D(1-v)\left[\frac{\partial}{\partial \mathbf{t}} \frac{\partial w}{\partial \mathbf{n}}+\kappa \frac{\partial w}{\partial \mathbf{t}}\right] \\
N=n_{i} m_{, i}=\frac{\partial m}{\partial \mathbf{n}}, \quad V=\frac{\partial m}{\partial \mathbf{n}}+\frac{\partial T}{\partial \mathbf{t}}
\end{gathered}
$$

where $\kappa$ is the curvature of the boundary contour and it is defined as

$$
\kappa:=n_{i} \frac{\partial t_{i}}{\partial \mathbf{t}}=-t_{i} \frac{\partial n_{i}}{\partial \mathbf{t}} \text {. }
$$

Now, let us consider separately particular cases of boundary conditions.

\subsection{Clamped edge}

Since the deflection is constant on the clamped and/or simply supported edge, we may write subsequently

$$
\left.\frac{\partial w}{\partial \mathbf{t}}\right|_{\Gamma}=0,\left.\quad \frac{\partial}{\partial \mathbf{t}} \frac{\partial w}{\partial \mathbf{t}}\right|_{\Gamma}=0
$$


Hence, in view of (8)-(10) and (5), we get

$$
T=-\left.D(1-v)\left[\frac{\partial}{\partial \mathbf{t}} \frac{\partial w}{\partial \mathbf{n}}+\kappa \frac{\partial w}{\partial \mathbf{t}}\right]\right|_{\Gamma}=0, \quad M=\left.m\right|_{\Gamma}, \quad N=\left.\frac{\partial m}{\partial \mathbf{n}}\right|_{\Gamma}=V
$$

\subsection{Simply supported edge}

Now, taking into account Eqs. (5), (12) and (8)-(10), we may write

$$
M=\left.m\right|_{\Gamma}-\left.\kappa D(1-v) \frac{\partial w}{\partial \mathbf{n}}\right|_{\Gamma}=0
$$

hence

$$
-\left.D(1-v) \frac{\partial}{\partial \mathbf{t}} \frac{\partial w}{\partial \mathbf{n}}\right|_{\Gamma}=-\left.\frac{1}{\kappa} \frac{\partial m}{\partial \mathbf{t}}\right|_{\Gamma}
$$

Finally, in view of (9) and (10), we get

$$
T=-\left.D(1-v) \frac{\partial}{\partial \mathbf{t}} \frac{\partial w}{\partial \mathbf{n}}\right|_{\Gamma}=-\left.\frac{1}{\kappa} \frac{\partial m}{\partial \mathbf{t}}\right|_{\Gamma}, \quad V=\left.\frac{\partial m}{\partial \mathbf{n}}\right|_{\Gamma}-\left.\frac{\partial}{\partial \mathbf{t}}\left(\frac{1}{\kappa} \frac{\partial m}{\partial \mathbf{t}}\right)\right|_{\Gamma}
$$

\subsection{Free edge}

In view of Eqs. (5) and (8)-(10), we obtain

$$
\begin{gathered}
M=\left.v m\right|_{\Gamma}-\left.D(1-v) \frac{\partial}{\partial \mathbf{n}} \frac{\partial w}{\partial \mathbf{n}}\right|_{\Gamma}=\left.m\right|_{\Gamma}-\left.D(1-v)\left[\kappa \frac{\partial w}{\partial \mathbf{n}}-\frac{\partial}{\partial \mathbf{t}} \frac{\partial w}{\partial \mathbf{t}}\right]\right|_{\Gamma}=0, \\
V=\left.\frac{\partial m}{\partial \mathbf{n}}\right|_{\Gamma}-\left.D(1-v) \frac{\partial}{\partial \mathbf{t}} \frac{\partial}{\partial \mathbf{n}} \frac{\partial w}{\partial \mathbf{t}}\right|_{\Gamma}=0
\end{gathered}
$$

Thus, having used the formulation with two field variables $\{w, m\}$, we can express the relevant physical boundary quantities $\{M, T, N, V\}$ in terms of the employed two field variables and/or their derivatives on the boundary edges. If the boundary edge is either clamped or simply supported, one does not need higher than first order derivatives of the field variables. Unfortunately, in the case of free edge, the second and third order derivatives of deflection are required in the expressions of the bending moment and generalized shear force, in general. Note that these higher order derivatives disappear even in the case of free edges, if the problem exhibits the translational symmetry along such an edge, when $\partial w / \partial \mathbf{t}=0$.

\section{Weak formulations on local sub-domains}

We will discuss two weak formulations corresponding to either one field variable $w(\mathbf{x})$ or two field variables $\{w(\mathbf{x}), m(\mathbf{x})\}$. 


\subsection{One field variable formulation}

The use of a constant test function in the weak form of the governing equation (1) on a local sub-domain $\Omega^{S}$ corresponds to the force equilibrium on $\Omega^{S}$

$$
\int_{\Omega^{s}}\left(D \nabla^{2} \nabla^{2} w-q\right) d \Omega=0 .
$$

Hence, one obtains immediately the local integral equation

$$
D \int_{\partial \Omega^{s}} n_{i}\left(\nabla^{2} w\right)_{, i} d \Gamma=\int_{\Omega^{s}} q d \Omega
$$

where the integrand on the left hand side is the transversal shear force on the boundary of the local sub-domain $\Omega^{S}$.

In order to consider the boundary conditions on the global boundary edge $\Gamma$, one needs the explicit expression of relevant boundary quantities $\{w, \partial w / \partial \mathbf{n}, M, V\}$ in terms of the deflection and its derivatives which are given by Eqs. (8)-(10). Note that the third order derivatives of the primary field variable $w$ are needed in both the LIE (18) and some of the boundary quantities.

\subsection{Two field variable formulation}

The weak forms of two governing equations (6) and (7) can be written as

$$
\begin{gathered}
D \int_{\partial \Omega^{s}} n_{i} w_{, i} d \Gamma+\int_{\Omega^{s}} m d \Omega=0 \\
\int_{\partial \Omega^{s}} n_{i} m_{, i} d \Gamma=-\int_{\Omega^{s}} q d \Omega
\end{gathered}
$$

The relevant boundary quantities $\{w, \partial w / \partial \mathbf{n}, M, V\}$ are expressed in terms of two field variables and their derivatives on boundary edges of particular kinds by Eqs. (13)-(17). Now, the first order derivatives of field variables are sufficient except the general case of free edges.

\section{Meshless approximations of field variables}

Two kinds of meshless approximations are used in this paper and shortly described in this section. The common notation $u(\mathbf{x})$ is employed for the scalar fields $w(\mathbf{x})$ and/or $m(\mathbf{x})$. The nodal points are freely distributed in the analyzed domain and on its boundary without creating any connectivity among the nodes. 


\subsection{Moving Least Square (MLS) approximation}

Recall, without going into details [3, 4], that the scalar field can be approximated in terms of certain nodal values $\hat{u}^{a}$ and shape functions $\phi^{g}(\mathbf{x})$ as

$$
u(\mathbf{x}) \approx \sum_{a=1}^{N^{q}} \hat{u}^{\bar{a}} \phi^{(q, a)}(\mathbf{x}), \quad \bar{a}=n(q, a)
$$

where $\bar{a}$ is the global number of the $a$-th node from the $N^{q}$ nodal points $\mathbf{x}^{\bar{a}} \in \mathcal{M}^{q}=\left\{\forall \mathbf{x}^{a} ; w^{a}\left(\mathbf{x}^{q}\right)>0\right\}_{a=1}^{N}$ with $\mathbf{x}^{q}$ being the central approximation node $(\mathrm{CAN}), w^{a}(\mathbf{x})$ is the weight function associated with the node $\mathbf{x}^{a}$, and $N$ is the total number of nodes. Recall that nodal unknowns $\hat{u}^{a}$ are certain expansion coefficients different from the nodal values of the approximated field. For the derivatives of the field variable, we shall use two approaches:

(i) Standard differentiation (DO)

$$
u_{, i}(\mathbf{x}) \approx \sum_{a=1}^{N^{q}} \hat{u}^{\bar{a}} \phi_{, i}^{(q, a)}(\mathbf{x}), \quad u_{, i j}(\mathbf{x}) \approx \sum_{a=1}^{N^{q}} \hat{u}^{\bar{a}} \phi_{, i j}^{(q, a)}(\mathbf{x}), \quad u_{, i j k}(\mathbf{x}) \approx \sum_{a=1}^{N^{q}} \hat{u}^{\bar{a}} \phi_{, i j k}^{(q, a)}(\mathbf{x})
$$

(ii) Modified differentiation (D1)

$$
u_{, i}(\mathbf{x}) \approx \sum_{a=1}^{N^{q}} \hat{u}_{i}^{\bar{a}} \phi^{(q, a)}(\mathbf{x}), \quad u_{, i j}(\mathbf{x}) \approx \sum_{a=1}^{N^{q}} \hat{u}_{i j}^{\bar{a}} \phi^{(q, a)}(\mathbf{x}), \quad u_{, i j k}(\mathbf{x}) \approx \sum_{a=1}^{N^{q}} \hat{u}_{i j k}^{\bar{a}} \phi^{(q, a)}(\mathbf{x})
$$

where

$$
\begin{aligned}
& \hat{u}_{i}^{d}=\sum_{g=1}^{N} G_{i}^{d g} \hat{u}^{g}, \quad \hat{u}_{i j}^{d}=\sum_{g, h=1}^{N} G_{j}^{d g} G_{i}^{g h} \hat{u}^{h}, \quad \hat{u}_{i j k}^{d}=\sum_{g, b, h=1}^{N} G_{k}^{d g} G_{j}^{g b} G_{i}^{b h} \hat{u}^{h}, \\
& G_{i}^{d g}:=\sum_{c=1}^{N}\left(E^{-1}\right)^{d c} F_{i}^{c g}, \quad E^{c d}:=\left\{\begin{array}{ll}
e^{c a}, & d=\bar{a} \\
0, & d \neq \bar{a}
\end{array}, \quad F_{i}^{c g}:=\left\{\begin{array}{cc}
f_{i}^{c a}, g=\bar{a} \\
0, & g \neq \bar{a}
\end{array},\right.\right.
\end{aligned}
$$

with $\bar{a}=n(c, a), a \in\left\{1,2, \ldots, N^{c}\right\}$ and $e^{c a}=\phi^{(c, a)}\left(\mathbf{x}^{c}\right), f_{i}^{c h}=\phi_{, i}^{(c, h)}\left(\mathbf{x}^{c}\right)$.

Note that in this approach we have used not higher than the first order derivatives of the shape functions.

\subsection{Point Interpolation Method (PIM)}

The utilization of the radial basis functions in combination with polynomials enables one to solve the problem of accuracy and numerical stability of the PIM- 
approximation $[5,6]$. Formally, the approximation takes the same form as in the MLS-approximation

$$
u(\mathbf{x}) \approx \sum_{a=1}^{N^{q}} u^{\bar{a}} \varphi^{(q, a)}(\mathbf{x}), \quad \bar{a}=n(q, a)
$$

but now $u^{g}$ stands for the nodal value of the approximated field variable, since $\varphi^{(q, a)}\left(\mathbf{x}^{\bar{b}}\right)=\delta_{a b}$. This difference is substantial also for the development of the modified scheme for calculation of derivatives of field variables. In D0approach, the standard derivatives of the field variable are given as

$$
u_{, i}(\mathbf{x}) \approx \sum_{a=1}^{N^{q}} u^{\bar{a}} \varphi_{, i}^{(q, a)}(\mathbf{x}), \quad u_{, i j}(\mathbf{x}) \approx \sum_{a=1}^{N^{q}} u^{\bar{a}} \varphi_{, i j}^{(q, a)}(\mathbf{x}), \quad u_{, i j k}(\mathbf{x}) \approx \sum_{a=1}^{N^{q}} u^{\bar{a}} \varphi_{, i j k}^{(q, a)}(\mathbf{x})
$$

while in D1-approach, we have

$$
u_{, i}(\mathbf{x}) \approx \sum_{a=1}^{N^{q}} u_{i}^{\bar{a}} \varphi^{(q, a)}(\mathbf{x}), \quad u_{, i j}(\mathbf{x}) \approx \sum_{a=1}^{N^{q}} u_{i j}^{\bar{a}} \varphi^{(q, a)}(\mathbf{x}), \quad u_{, i j k}(\mathbf{x}) \approx \sum_{a=1}^{N^{q}} u_{i j}^{\bar{a}} \varphi_{, k}^{(q, a)}(\mathbf{x})
$$

where

$$
u_{i}^{c}=\sum_{a=1}^{N^{c}} f_{i}^{c a} u^{\bar{a}}, \quad u_{i j}^{c}=\sum_{a=1}^{N^{c}} f_{j}^{c a} \sum_{g=1}^{N^{\bar{a}}} f_{i}^{\bar{a} g} u^{\bar{g}}, \quad u_{i j k}^{c}=\sum_{a=1}^{N^{c}} f_{k}^{c a} \sum_{g=1}^{N^{\bar{a}}} f_{j}^{\bar{a} g} \sum_{h=1}^{N^{\bar{g}}} f_{i}^{\bar{g} h} u^{\bar{h}},
$$

with $f_{i}^{c a}:=\varphi_{, i}^{(c, a)}\left(\mathbf{x}^{c}\right)$.

In comparison with the MLS-approximation, the decrease of the efficiency of the numerical computations due to utilization of D1-approach is less expressive, since it is sufficient to pre-compute only the matrix $f_{i}^{c g}$ instead of matrices $E^{c d},\left(E^{-1}\right)^{d c}, F_{i}^{c g}$.

\section{Rotationally symmetric bending of circular plates}

Because of the symmetry, the considered problem is simplified when polar coordinates $(r, \varphi)$ are employed instead of Cartesian coordinates and $\partial(.) / \partial \varphi \equiv 0$ Then, the governing equation becomes

$$
\begin{gathered}
D\left(\frac{\partial^{2}}{\partial r^{2}}+\frac{1}{r} \frac{\partial}{\partial r}\right)^{2} w(r)=q(r) \\
\text { and } M=-\left.D\left[(1-v) \frac{\partial^{2} w(r)}{\partial r^{2}}+v \nabla^{2} w(r)\right]\right|_{\Gamma}, \quad T \equiv 0, \quad V=N=\left.\mp D \frac{\partial \nabla^{2} w(r)}{\partial r}\right|_{\Gamma} .
\end{gathered}
$$


The weak form of equation (23) on the local sub-domain $\Omega^{c}$ takes the form

$$
\left.D\left(r \frac{\partial^{3} w(r)}{\partial r^{3}}+\frac{\partial^{2} w(r)}{\partial r^{2}}-\frac{1}{r} \frac{\partial w(r)}{\partial r}\right)\right|_{r^{c}-r_{o}} ^{r^{c}+r_{o}}=\int_{r^{c}-r_{o}}^{r^{c}+r_{o}} r q(r) d r
$$

since $\Omega^{c}=\left\{\forall(r, \varphi) \in \Omega ; r \in\left[r^{c}-r_{0}, r^{c}+r_{0}\right], \varphi \in[0,2 \pi]\right\}$.

In the case of two field variables formulation, the governing equations become

$$
D \frac{1}{r} \frac{\partial}{\partial r}\left(r \frac{\partial w(r)}{\partial r}\right)+m(r)=0, \quad \frac{1}{r} \frac{\partial}{\partial r}\left(r \frac{\partial m(r)}{\partial r}\right)=-q(r)
$$

and the weak form on local sub-domain $\Omega^{c}$ is given as

$$
\left.D r \frac{\partial w(r)}{\partial r}\right|_{r^{c}-r_{o}} ^{r^{c}+r_{o}}+\int_{r^{c}-r_{o}}^{r^{c}+r_{o}} r m(r) d r=0,\left.\quad r \frac{\partial m(r)}{\partial r}\right|_{r^{c}-r_{o}} ^{r^{c}+r_{o}}=-\int_{r^{c}-r_{o}}^{r^{c}+r_{o}} r q(r) d r .
$$

Since

$$
M=\left.\left[m(r)+D(1-v) \frac{1}{r} \frac{\partial w(r)}{\partial r}\right]\right|_{\Gamma}, T=0, \quad N= \pm\left.\frac{\partial m(r)}{\partial r}\right|_{\Gamma}=V
$$

the weak form of the two field variable formulation does not involve higher than the first order derivatives of the field variables. On the other hand, the highest order of the derivatives is equal to two in the strong form given by Eq. (25).

\section{Numerical examples}

Several numerical examples will be considered for rotationally symmetric bending of circular plate for which exact solutions are available. Then, we can investigate the accuracy and convergence as well as computational efficiency of various presented formulations and techniques. The accuracy of numerical solutions of boundary value problems will be characterized by global error norm determined by squared deviations of the computed nodal values from the exact values of the field variables. In all numerical computations, we have used a uniform distribution of nodal points and the radius of the sub-domain $r_{o}=0.1 h$ with $h$ being the distance between two neighbour nodes. The following notations LIE(biharm), LIE(2xPoiss), CPDE(2xPoiss) are employed for the numerical results obtained by the weak formulation of the biharmonic problem (one field formulation), weak and strong formulation for decomposed problems (two field formulation), respectively. 
We present the numerical results for three b.v.p.: (A) $r \in\left[0, r_{a}\right]$, clamped edge (CE) $r=r_{a}$; (B) $r \in\left[0, r_{a}\right]$, simply supported edge (SSE) $r=r_{a}$; (C) $r \in\left[r_{b}, r_{a}\right]$ simply supported edge (SSE) $r=r_{b}$, free edge $r=r_{a}$.

The accuracy and convergence for numerical solutions of three considered b.v.p. are presented in Fig. 1. It can be seen that only the formulations for decomposed problem yield meaningful results. In the LIE(2xPoiss) formulation, the D1-approach is identical with the D0-approach, since not higher that the $1^{\text {st }}$ order derivative enter the formulation. Note that the D1-approach yields slightly better convergence of accuracy in the $\mathrm{CPDE}(2 \mathrm{xPoiss})$ than the $\mathrm{D} 0$-approach.
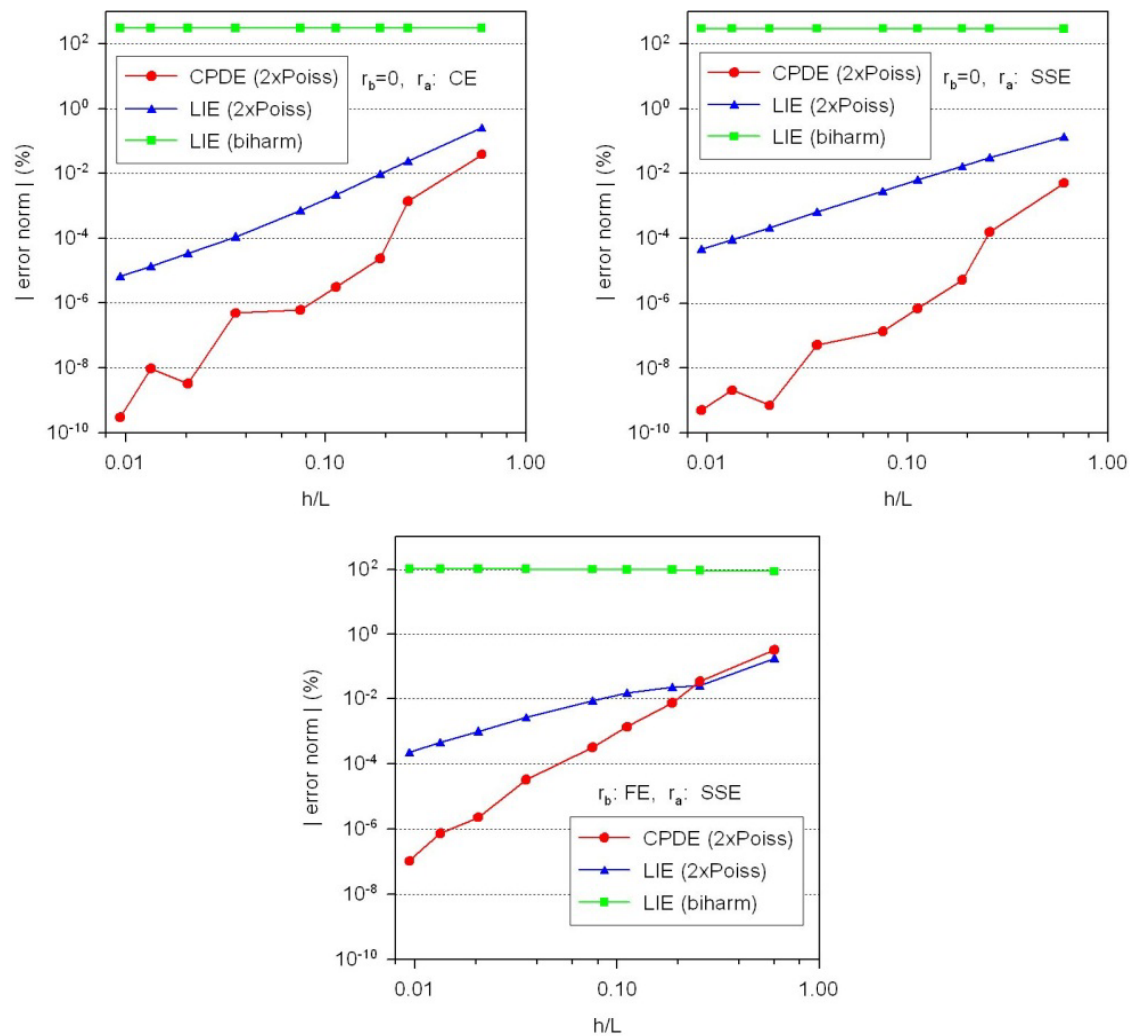

Figure 1: Comparison of accuracy and convergence of numerical solutions for three kinds of b.v.p. by three formulations combined with D1approach within MLS-approximations of field variables.

Similar analysis has been carried out also with using the PIM-approximation (see Fig. 2). The LIE(biharm) formulation gives unreliable results (convergence is achieved only in the b.v.p. (C) ). Both the LIE(2xPoiss) and CPDE(2xPoiss) formulations give stable and highly accurate numerical solutions of the b.v.p. (A) 
and (B); in the case of b.v.p. (C) the accuracy is good with excellent convergence rate. Note that the D1-approach slightly improves the accuracy of numerical solutions by the CPDE(2xPoiss) formulation as compared with using D0approach for approximation of derivatives of field variables.
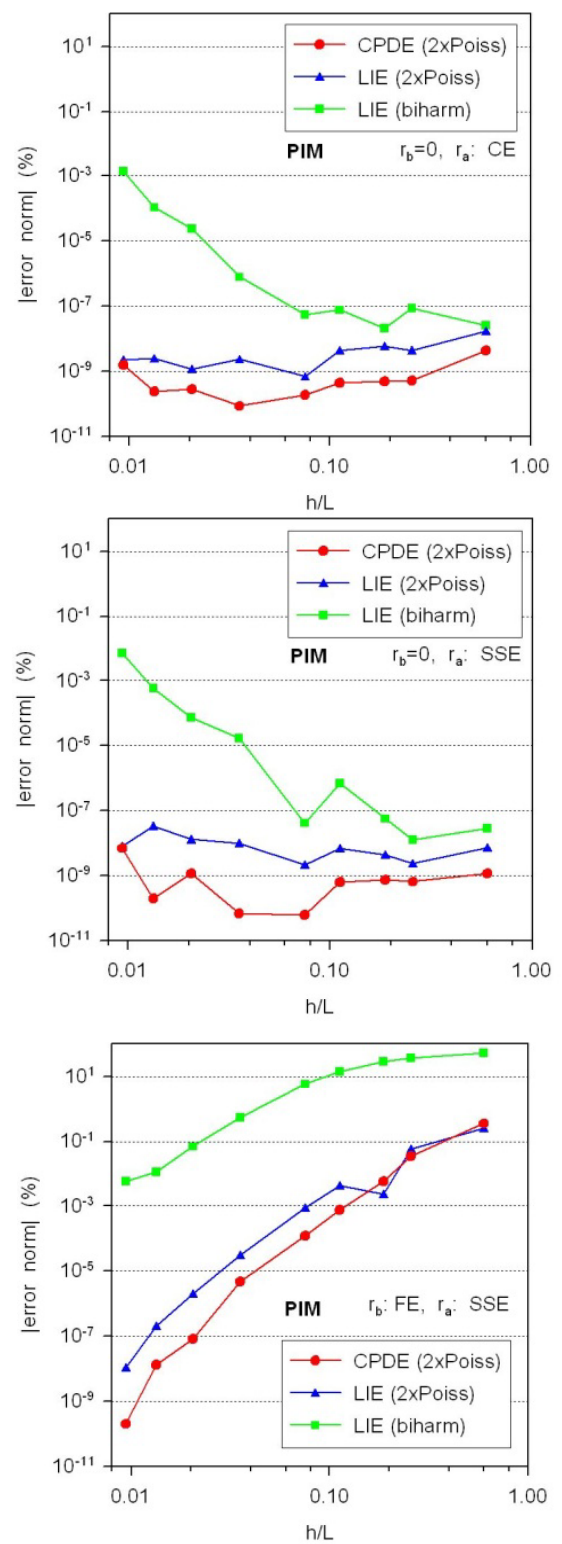

Figure 2: Comparison of accuracy and convergence of numerical solutions for three kinds of b.v.p. by three formulations combined with D1approach within PIM-approximations of field variables. 
It is interesting to compare the computational efficiency of the discussed methods (Fig. 3). It can be seen that the D1-approach gives rise to a substantial prolongation of the CPU-time when a large amount of nodes in the CPDE(2xPoiss) and/or LIE(biharm) formulations is employed. Note that the increase of the CPU-time in the case of PIM-approximation is less expressive than in the case MLS-approximation, where certain inverse matrices are required in the D1-approach. The increase of the CPU-time due to utilization of D1approach is inadequate price for improvement of accuracy by $\operatorname{LIE}(2 \times P o i s s)$ formulation.
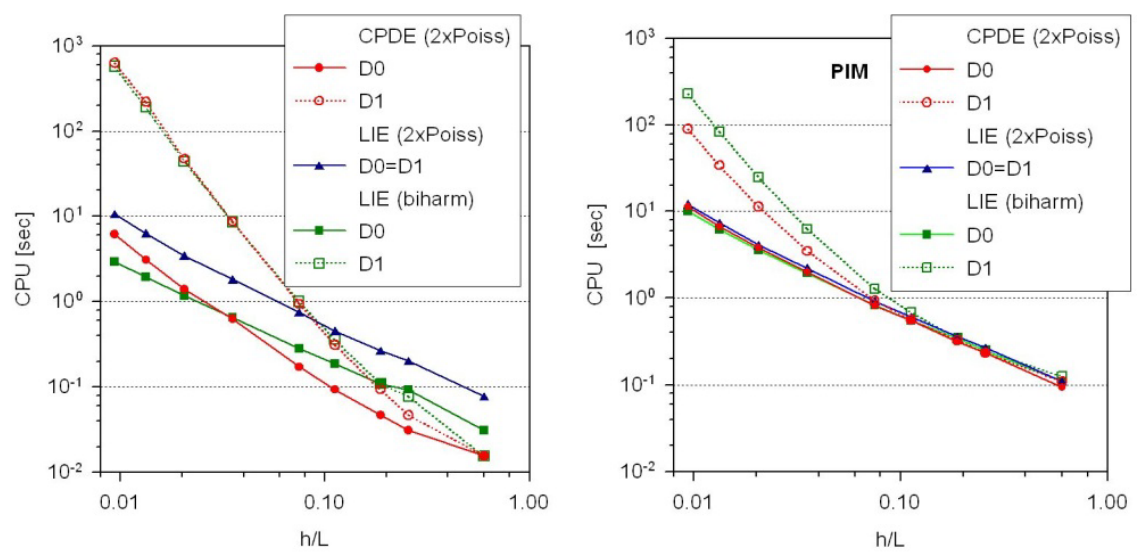

Figure 3: Comparison of computational times spent by various formulations implemented by PIM- and/or MLS-approximation.

\section{Conclusions}

The decomposition of the thin plate bending problems governed by the biharmonic operator into two coupled problems governed by Poisson equations is developed and discussed. Two kinds of meshless approximations of field variables are employed in each formulation for numerical solution of boundary value problems. In contrast to the boundary element formulation for decomposed problem, all the physical quantities occurring in the possible boundary conditions can be expressed in terms of the field variables and their derivatives. Thus, the developed decomposed formulations are not restricted to certain class of boundary value problems. For solution of the original biharmonic problem, we have developed only the weak formulation, in order to avoid the fourth order derivatives in the strong formulation. For solution of the decomposed problem, both the strong and local weak formulations have been developed. The accuracy, convergence of accuracy and computational efficiency have been studied for three formulations combined with two meshless approximation of field variables in simple boundary value problems for circular plate. The discussed methods give reasonable numerical results when applied to decomposed problem, while 
the methods applied to original biharmonic problem fail. The accuracy can be further improved by introducing a modified differentiation technique for approximation of the derivatives of field variables, but it leads to an enormous increase of the computational time.

\section{Acknowledgement}

This work was partially supported by the Slovak Research and Development Agency under the contract No. APVV-0032-10.

\section{References}

[1] Timoshenko, S.P., Woinowsky-Krieger, S., Theory of Plates and Shells, McGraw-Hill: New York, 1959.

[2] Atluri, S.N., Shen, S., Simulation of a $4^{\text {th }}$ order ODE: Illustration of various primal and mixed MLPG methods. CMES - Computer Modeling in Engineering and Sciences, 7, pp. 241-268, 2005.

[3] Lancaster, P., Salkauskas, K., Surfaces generated by moving least square method. Math. Comput., 37, pp. 141-158, 1981.

[4] Sladek, V., Sladek, J., Zhang, Ch., Computation of stresses in nonhomogeneous elastic solids by local integral equation method: a comparative study. Computational Mechanics. 41, pp. 827-845, 2008.

[5] Liu, G.R., Mesh Free Methods, Moving Beyond the Finite Element Method, CRC Press: Boca Raton, 2003.

[6] Sladek, V., Sladek, J., Tanaka, M., Local integral equations and two meshelss interpolations with application to potential problems in nonhomogeneous media. CMES -Computer Modeling in Engineering and Sciences, 7, pp. 69-83, 2005. 